\title{
A Phenotypical Case of Anti-MDA5 Positive Dermatomyositis
}

\author{
Kelly B Scarberry ${ }^{1}$, Akaolisa Eziokwu², Mohammad Khan ${ }^{2}$ and Mohit Gupta ${ }^{2 *}$ \\ ${ }^{1}$ Case Western Reserve University School of Medicine, Cleveland, Ohio, USA \\ ${ }^{2}$ Cleveland Clinic, Medicine Institute, Cleveland, Ohio, USA
}

\begin{abstract}
Introduction: Melanoma differentiation-associated protein 5 (MDA5) antibody-positive dermatomyositis displays unique cutaneous and pathologic features.

Case Summary: A 71-year old woman developed a periorbital heliotrope rash, painful palmar papules, and oral ulcerations. Months later, she presented with exertional oxygen desaturation and computed tomography of her chest demonstrated new ground-glass opacities present. Antibodies to MDA5 were present in her serum.

Discussion: MDA5-positive dermatomyositis is characterized by amyopathic disease with rapidly progressively interstitial lung disease. As seen in this patient, the mucocutaneous symptoms may precede the onset of lung disease and are crucial in identifying those at risk.
\end{abstract}

Abbreviations: Melanoma differentiation-associated protein: 5MDA5

\section{Introduction}

Dermatomyositis is an idiopathic autoimmune disease typically characterized by symmetric proximal muscle weakness, muscle inflammation, and skin manifestations [1]. Diagnosis is based on clinical features of typical cutaneous findings (e.g. Gottron's papules and heliotrope rash) and proximal muscle involvement, laboratory evidence of muscle damage, pathologic correlation on biopsy, and identification of myositis-specific autoantibodies. Myositis-specific autoantibodies are found in $50 \%$ to $70 \%$ of dermatomyositis patients and clinically distinct phenotypes have been described for different autoantibodies [2,3] This report describes a case of dermatomyositis with seropositivity to the autoantigen melanoma differentiationassociated protein 5 (MDA5). The phenotype of anti-MDA5 positive dermatomyositis displays unique cutaneous and pathologic features and is of prognostic importance.

\section{Case Presentation}

A 71-year old woman presented to her doctor with a right periorbital erythematous, scaly rash (Figure 1). She was initially treated with several rounds of antibiotics for presumed blepharitis with little improvement. She then developed painful, erythematous papules on her palms and on the ventral and lateral aspect of her fingers (Figure 2). She also developed recurrent oral mucosal pain and ulceration of her gingiva and tongue. Two months following initial appearance of the eye rash, she experienced mild symmetric polyarthralgias. She subsequently underwent a full rheumatologic workup that was negative for antinuclear antibodies, antineutrophil cytoplasmic antibodies, rheumatoid factor, and anti-dsDNA. She also had a negative extractable nuclear antigen panel, including anti-Jo-1 and anti-Mi-2. Serum creatine kinase and aldolase levels were within normal limits.
Months later, the patient then developed a dry cough and shortness of breath on exertion and initially received antibiotics for communityacquired pneumonia. She was subsequently hospitalized due to persistent symptoms. She was discharged and outpatient pulmonary function tests were performed, which were consistent with a restrictive lung disease. She was readmitted to the hospital for exertional oxygen desaturation and CT-chest without contrast revealed new, diffuse bilateral ground glass opacities that were not present two months prior, suggestive of rapidly-progressive interstitial lung disease. An extended myositis panel tested positive for anti-MDA5 antibody. She was treated with prednisone followed by maintenance mycophenolate mofetil and cyclophosphamide. Following treatment, the patient's pulmonary symptoms improved; however, follow-up pulmonary function testing four months later remained unchanged.

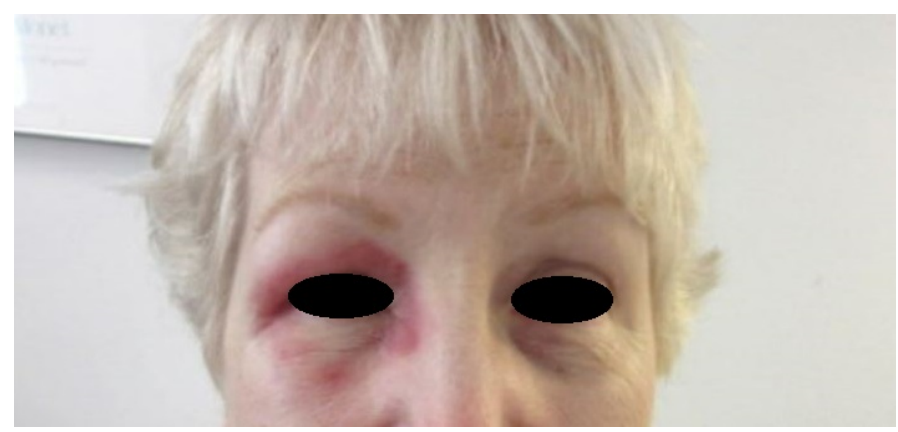

Figure 1. Violaceous heliotrope rash of right periorbital area

${ }^{*}$ Correspondence to: Mohit Gupta, Department of Internal Medicine, Cleveland clinic, Ohio-44195, USA, Tel: +1(216)401-6104; E-mail: GUPTAM32@ccf.org

Key words: dermatomyositis, interstitial lung disease, case report

Received: July 18, 2018; Accepted: July 26, 2018; Published: July 28, 2018 


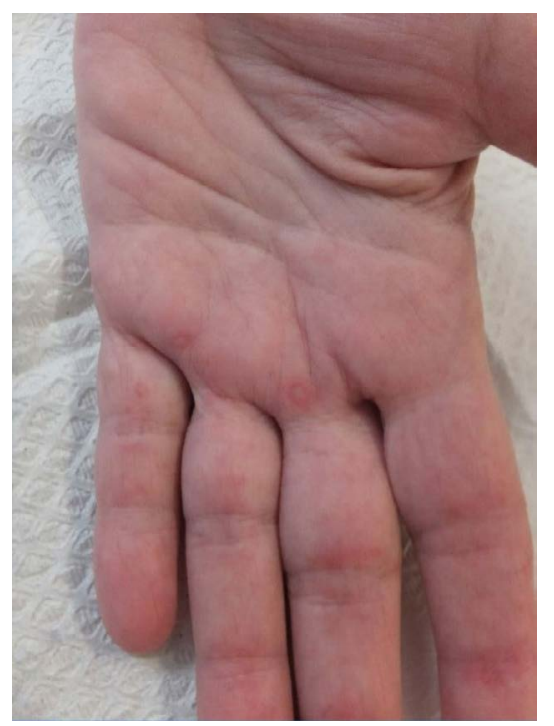

Figure 2. Small erythematous papules on palm and fingers of left hand

\section{Discussion}

As seen in this patient, MDA5 autoantibodies are associated with clinically amyopathic dermatomyositis [4]. Anti-MDA5 dermatomyositis patients tend to have no muscle weakness or laboratory evidence of muscle damage, although some will have elevated muscle enzymes without clinically apparent weakness [5]. Anti-MDA5 dermatomyositis is also associated with rapidly-progressive interstitial lung disease and portends an unfavorable prognosis [6]. The progressive lung disease is typically poorly responsive to immunosuppressive therapy. Serum levels of MDA5 antibodies correlate with severity of lung disease and are predictive of outcomes [7].

Dermatomyositis is also associated with many skin changes, including an erythematous to violaceous heliotrope rash of the periorbital region and Gottron's papules over the dorsum of metacarpophalangeal and interphalangeal joints [1]. Painful palmar papules, oral pain, and ulceration are mucocutaneous findings that are associated with anti-MDA5 dermatomyositis [8] and were present in our patient. The case presented here found that mucocutaneous manifestations of anti-MDA5 dermatomyositis may manifest before the onset of lung disease. Presence of these findings warrant testing for MDA5 autoantibodies. Increased awareness of the clinical phenotype is needed, as early recognition is critical to treating the associated rapidly-progressive interstitial lung disease.

\section{Funding}

\section{None}

\section{Interests}

The authors declare they have no competing interests.

\section{References}

1. Dalakas MC, Hohlfeld R (2003) Polymyositis and dermatomyositis. Lancet 362: 971982. [Crossref]

2. Plotz PH, Dalakas M, Leff RL (1989) Current concepts in the idiopathic inflammatory myopathies: polymyositis, dermatomyositis, and related disorders. Ann Intern Med 111:143.

3. Levine SM, Raben N, Xie D (2007) Novel conformation of histidyl-transfer RNA synthetase in the lung: the target tissue in Jo-1 autoantibody-associated myositis. Arthritis Rheum 56: 2729-2739.

4. Sato S, Hoshino K, Satoh T (2009) RNA helicase encoded by melanoma differentiationassociated gene 5 is a major autoantigen in patients with clinically amyopathic dermatomyositis: association with rapidly progressive interstitial lung disease. Arthritis Rheum 60: 2193-2200.

5. Sato S, Hirakata M, Kuwana M (2005) Autoantibodies to a 140-kd polypeptide, CADM-140, in Japanese patients with clinically amyopathic dermatomyositis. Arthritis Rheum 52: 1571-1578

6. Labrador-Horrillo M, Martinez MA, Selva-O'Callaghan A (2014) Anti-MDA5 Antibodies in a Large Mediterranean Population of Adults with Dermatomyositis. $J$ Immunol Res pp. 1-8.

7. Sato S, Masataka K, Fujita T (2013) Anti-CADM-140/MDA5 autoantibody titer correlates with disease activity and predicts disease outcome in patients with dermatomyositis and rapidly progressive interstitial lung disease. Mod Rheumatol 3: 496-502.

8. Fiorentino D, Chung L, Zwerner J, Rosen A, Casciola-Rosen L (2011) The mucocutaneous and systemic phenotype of dermatomyositis patients with antibodies to MDA5 (CADM-140): a retrospective study. J Am Acad Dermatol 65: 25-34. [Crossref]

Copyright: $@ 2018$ Scarberry KB. This is an open-access article distributed under the terms of the Creative Commons Attribution License, which permits unrestricted use, distribution, and reproduction in any medium, provided the original author and source are credited. 